\title{
Doctor, me duelen las muñecas
}

\author{
Amparo Manzano Juárez ${ }^{a}$, Salima Qudsi Sinclair ${ }^{b}$, María Dolores González Céspedes ${ }^{c}$ \\ y Marina Manuela Antonio González ${ }^{\mathrm{d}}$.
}

\author{
${ }^{a}$ M.I.R. Medicina de Familia y \\ Comunitaria. Centro de Salud \\ Hellín II. Albacete (España). \\ b Médico de Medicina Física \\ y Rehabilitación. Hospital de \\ Hellín. Albacete (España). \\ ${ }^{c}$ Médico de Familia. Centro \\ de Salud Hellín II. Albacete \\ (España). \\ ${ }^{d}$ Médico de Urgencias. Hospital \\ de Cieza. Murcia (España).
}

Correspondencia:

Amparo Manzano Juárez.

C/ Jacinto Benavente 19, $4^{\circ} \mathrm{V}$. C.P. 02400. Hellín (Albacete). España.

Correo electrónico: ampamanjua@hotmail.com.

Recibido el 26 de septiembre de 2014.

Aceptado para su publicación el 23 de octubre de 2014.

\begin{abstract}
RESUMEN
Presentamos el caso de una mujer de 40 años con dolor en ambas muñecas que aparece de forma progresiva y de meses de evolución. Se acompaña de parestesias, probablemente por atrapamiento del nervio mediano. Un adecuado diagnóstico diferencial incluye las patología congénitas y adquiridas. Tras la exploración física y hallazgos radiológicos se llega al juicio clínico final.
\end{abstract}

Palabras Clave: Trastornos del Crecimiento. Lesiones de la Muñeca. Parestesia.

\section{ABSTRACT}

Doctor, my wrists hurt

We present the case of a 40 year-old woman with progressive pain in both wrists of months of evolution, and accompanied by paresthesia, probably due to median nerve entrapment. An appropriate differential diagnosis includes both congenital and acquired pathologies. Final diagnosis is reached after physical examination and radiological findings.

Key words: Growth Disorders. Wrist Injuries. Paresthesia.

\section{INTRODUCCIÓN}

La deformidad de Madelung es un trastorno congénito raro del carpo caracterizado por un crecimiento asimétrico y una curvatura de la fisis del radio distal. Se produce un cierre prematuro epifisario en su porción medial y palmar anterior del radio distal, produciendo un acortamiento del mismo y un aparente sobrecrecimiento del cúbito ${ }^{1-3}$. Se asocia frecuentemente a síndromes genéticos como la discondrosteosis de Léri-Weill y el síndrome de Turner. Alteraciones similares a las producidas en la deformidad de Madelung las encontramos en otras patologías como tumores, traumatismos, displasias óseas generalizadas, infecciones y endocrinopatías ${ }^{2,4}$. Estas enfermedades se deben tener en cuenta a la hora de hacer el diagnóstico definitivo. Por tanto, ante la presencia de deformidad y dolor en la muñeca hay que hacer un buen diagnóstico diferencial.

\section{CASO CLÍNICO}

Mujer de 40 años que acude a la consulta de Atención Primaria por dolor en ambas muñecas, pérdida de fuerza en las manos y parestesias en todos los dedos, de mayor frecuencia e intensidad en la mano derecha. La paciente presentaba un dolor crónico de cinco meses de evolución de características mecánicas y empeoramiento progresivo, asociando una deformidad de las 
muñecas. No ha existido ningún traumatismo previo que justifique la presencia de la clínica referida. No presenta antecedentes patológicos de interés. Es diestra y trabaja cuidando ancianos.

A la exploración física de ambas muñecas destaca la presencia de una deformidad en la cara dorsal a nivel del estiloides cubital, asociada a una desviación cubital. Además, se objetiva un aumento de la sudoración y enrojecimiento de las manos.

El balance articular (BA) de las muñecas era simétrico: Flexión dorsal: $40^{\circ}$. Flexión palmar: $55^{\circ}$. Desviación radial: $20^{\circ}$. Desviación cubital: $30^{\circ}$. Supinación: faltan los últimos grados. Pronación: completa.
Presenta dolor a la palpación a nivel de la articulación radio-carpiana distal y molestias a la palpación de la cabeza del radio. Muestra un BA de codo completo. El balance muscular (BM) flexoextensión de las muñecas es 4/5.

Por otra parte, la presa-puño-pinza es completa. La oposición del pulgar está conservada. Las articulaciones metacarpo-falángicas, interfalángicas proximales e interfalángicas distales están libres. Presenta un signo de Tinnel mediano negativo y un signo de Phalen mediano dudoso.

Ante los hallazgos clínicos descritos, se solicita una radiografía anteroposterior y lateral de ambas muñecas (figuras 1 y 2).

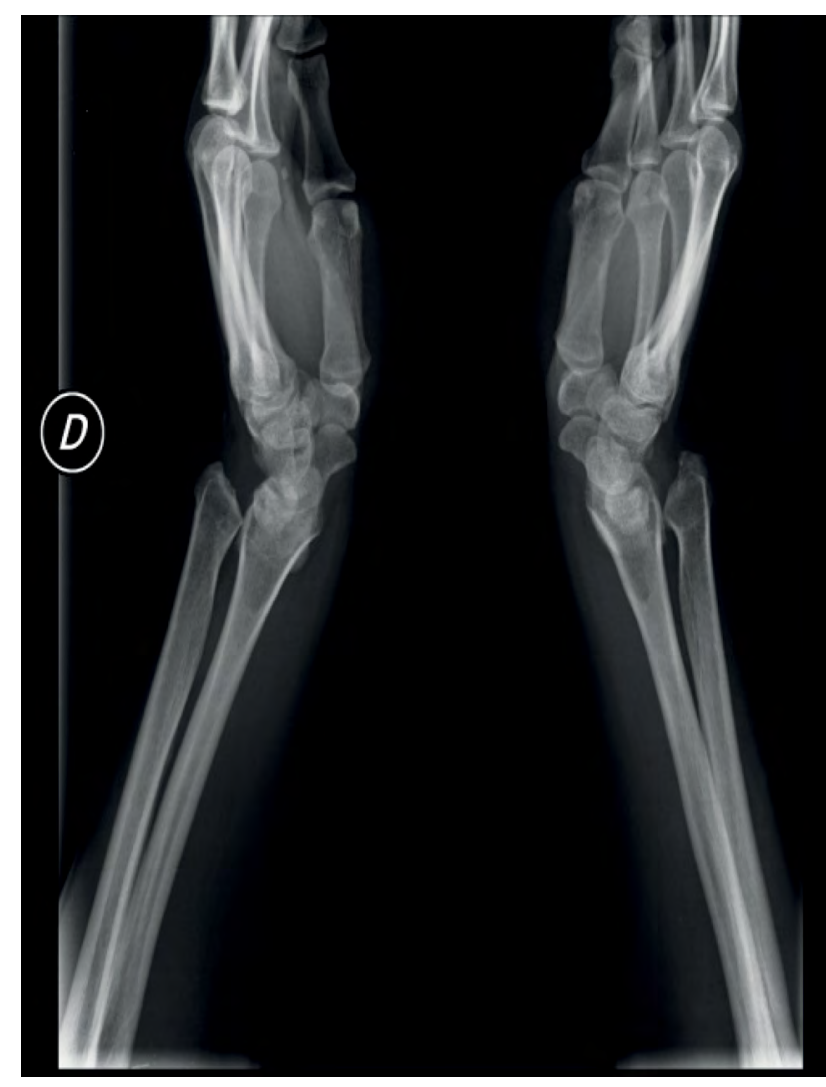

Figura 1. Radiografía lateral de ambas muñecas. Se observa luxación cubital.

Tras objetivar la presencia de deformidad en la radiografía se deriva a consultas externas de Traumatología para valoración, donde se realiza el diagnóstico de deformidad de Madelung y luxación cubital distal bilateral.

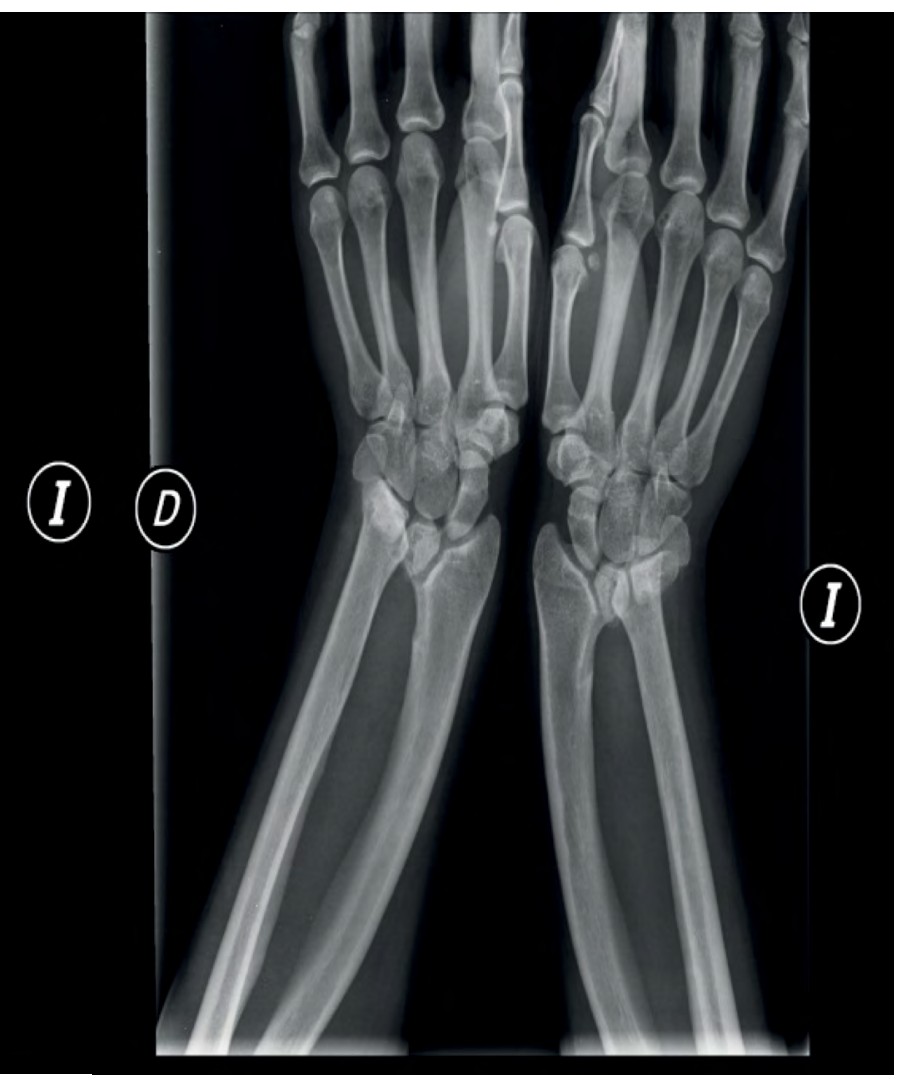

Figura 2. Radiografía anteroposterior de ambas muñecas. Se observa angulación y acortamiento del radio distal bilateral.

Se propone tratamiento quirúrgico de reconstrucción del carpo, pero la paciente lo rechaza, por lo que se realiza interconsulta a Rehabilitación. Pautan férula posicional bilateral nocturna más tratamiento de fisioterapia (tonificación musculatura intrínseca de mano y electroterapia analgésica). 


\section{DISCUSIÓN}

La deformidad de Madelung típicamente se presenta de forma bilateral como una tumoración de lento crecimiento a nivel dorsocubital de las muñecas, acompañada de dolor local y disminución de la movilidad. Normalmente se manifiesta antes de los 20 años, siendo más frecuente en el sexo femenino. Los pacientes se encuentran limitados para la pronación y supinación del carpo afecto. Los casos graves desarrollan artrosis progresiva e inestabilidad de la articulación radiocubital distal, artrosis radiocarpiana, y pueden complicarse con rotura de los tendones extensores de los dedos ${ }^{1,3}$.

En estos pacientes se debe realizar una historia clínica detallada, y solicitar las pruebas complementarias necesarias que nos ayuden a realizar el diagnóstico definitivo, y descartar otras patologías de características similares.

En nuestro caso la paciente no refiere antecedente traumático, cuadro constitucional, ni signos de infección (fiebre, tumefacción, etc.) o afectación a otro nivel articular que pudiera sugerir otro tipo de enfermedad.

Considerando las manifestaciones clínicas (dolor crónico de evolución progresiva, deformidad y simetría), se solicitó una radiografía simple antero- posterior y lateral bilateral que permitió realizar el diagnóstico definitivo.

No existen guías de tratamiento específico. Al inicio de la enfermedad se recomienda utilizar tratamiento conservador con analgésicos, antiinflamatorios no esteroideos y férulas para evitar la excesiva movilización de articulación. El tratamiento quirúrgico se decide en función de cuatro factores: edad de la paciente y crecimiento previsible del radio distal, gravedad de la deformidad, severidad de síntomas y los hallazgos clínicos-radiológicos. El objetivo quirúrgico es aliviar el dolor, la corrección estética y mejorar la movilidad articular ${ }^{1-4}$.

\section{BIBLIOGRAFÍA}

1. Ly-Pen D, Andreu JL. Deformidad de Madelung. Reumatol Clin. 2014; 10 (2): 125-6.

2. Sifuentes Giraldo WA, Gámir Gámir ML. Acquired Madelung's deformity in rheumatoid factor-positive polyarticular juvenile idiopathic arthritis. Reumatol Clin. 2014; 10 (4): 266-7.

3. Ghatan AC, Hanel DP. Madelung deformity. J Am Acad Orthop Surg. 2013 Jun; 21 (6): 372-82.

4. Kumar A, Rai GK, Akhtar J, Phillip R, Gutch M, Arya TV. All Madelung deformities are not endocrine. Indian J Endocrinol Metab. 2013; 17 (Suppl1): S231-3. 